\title{
Pelvic inflammatory disease and salpingitis: incidence of primary and repeat episodes in England
}

\author{
M. J. PRICE ${ }^{1 *}$, A. E. ADES ${ }^{2}$, N. J. WELTON ${ }^{2}$, I. SIMMS ${ }^{3}$ AND P. J. HORNER ${ }^{2,4}$ \\ ${ }^{1}$ Institute of Applied Health Research, University of Birmingham, Birmingham, UK \\ ${ }^{2}$ School of Social and Community Medicine, University of Bristol, Bristol, UK \\ ${ }^{3}$ Health Protection Agency, Colindale, London, UK \\ ${ }^{4}$ Bristol Sexual Health Centre, University Hospital Bristol NHS Foundation Trust, Bristol, UK
}

Received 1 June 2016; Accepted 17 August 2016;

first published online 28 September 2016

\section{SUMMARY}

Pelvic inflammatory disease (PID) and more specifically salpingitis (visually confirmed inflammation) is the primary cause of tubal factor infertility and is an important risk factor for ectopic pregnancy. The risk of these outcomes increases following repeated episodes of PID. We developed a homogenous discrete-time Markov model for the distribution of PID history in the UK. We used a Bayesian framework to fully propagate parameter uncertainty into the model outputs. We estimated the model parameters from routine data, prospective studies, and other sources. We estimated that for women aged $35-44$ years, 33.6\% and $16 \cdot 1 \%$ have experienced at least one episode of PID and salpingitis, respectively (diagnosed or not) and 10.7\% have experienced one salpingitis and no further PID episodes, $3.7 \%$ one salpingitis and one further PID episode, and $1 \cdot 7 \%$ one salpingitis and $\geqslant 2$ further PID episodes. Results are consistent with numerous external data sources, but not all. Studies of the proportion of PID that is diagnosed, and the proportion of PIDs that are salpingitis together with the severity distribution in different diagnostic settings and of overlap between routine data sources of PID would be valuable.

Key words: Cumulative incidence, incidence, Markov model, pelvic inflammatory disease (PID), repeat PID.

\section{INTRODUCTION}

Pelvic inflammatory disease (PID) is the primary cause of tubal factor infertility (TFI) and an important risk factor for ectopic pregnancy (EP). It comprises a spectrum of upper genital tract inflammatory disorders in women, which includes any combination of endometritis, salpingitis, tubo-ovarian abscess and pelvic peritonitis [1]. PID is difficult to diagnose and the criteria for a clinical diagnosis of PID have changed over time with

\footnotetext{
* Author for correspondence: Dr M. J. Price, University of Birmingham, School of Health and Population Sciences, Public Health Building, Edgbaston, Birmingham B15 2TT, UK. (Email: m.price.2@bham.ac.uk)
}

the recognition that atypical milder clinical manifestations are common [2,3], but may still be associated with reproductive damage. In UK national PID guideline 2011, recent onset of lower abdominal pain in association with local tenderness on bimanual examination is considered sufficient to establish a diagnosis and initiate treatment [4]. Clinical information can also be used to classify PID as 'possible', 'probable' and 'definite' PID based on Hager's criteria [5, 6]. This classification is often used in clinical trials (e.g. POPI [7]) and in studies of patients' data, such as the General Practice Research Database (GPRD) [6-8].

Over $50 \%$ of diagnosed PID episodes in England are treated in primary care, the remainder being 
treated in sexually transmitted infection (STI) clinics or hospital. Diagnosed PID underestimates true PID incidence as a relatively high proportion of PID is undiagnosed because of the range of clinical manifestations and difficulty in making a diagnosis [3, 9]. However, a cross-sectional study found that while $66 \%$ of TFI cases reported no previous diagnosis of PID, only $11 \%$ reported never having had clinical symptoms [10]; suggesting that while a large proportion of the PID that causes TFI is undiagnosed it usually is not completely asymptomatic.

PID is mostly caused by STIs, such as Chlamydia trachomatis $(\mathrm{CT})$, gonorrhoea, or bacterial vaginosisassociated microbes; by respiratory and enteric pathogens that colonize the female genital tract, and, more rarely, infections introduced during surgery, abortion, or parturition $[3,11]$.

Much of our knowledge of the impact of PID on reproductive health is based on the Lund study [2, 12-14]. The study was based on laparoscopic examination of women with hospital-diagnosed PID. Women with clinical PID but with no salpingitis, defined as visible inflammation of the fallopian tubes, experienced EP and TFI at no more than the background rate. Incidence of EP and TFI in women with salpingitis depends on age and severity of index salpingitis, and increases markedly with the number of subsequent PID episodes.

On the basis of these findings, the present study sets out to provide estimates of population-level age-specific mean incidence of PID and salpingitis, with a particular focus on the incidence of repeat episodes, as these appear specifically associated with poor reproductive outcomes. Our primary interest lies in natural history in the absence of screening for STI infections so we used data for 2002. We developed a homogenous Markov model to describe PID and salpingitis history in women in England. The estimates offer opportunities to validate risk estimates for infections that cause PID, and provide estimates of cumulative exposure for population attributable fraction calculations. Furthermore, estimates of the exposure distribution allow results from cohort studies following patients with PID/salpingitis to be validated against population sequelae estimates. We estimated the model parameters using a variety of data sources and methods and validated the model against a number of external data sources.

\section{MATERIALS AND METHODS}

We developed an 8-state discrete-time homogenous Markov structure with 1-year cycles to describe the distribution of PID and salpingitis (Fig. 1). The model is designed to estimate not only the cumulative incidence of episodes, but also the age-specific proportions of the population who have experienced $1,2, \geqslant 3$ episodes. Separate estimations are generated for three kinds of 'episode': PID, salpingitis, and PID in women who have experienced salpingitis.

We assumed that women who have an episode of the type(s) specified in the model have a different (higher) rate of subsequent episodes for the next 2 years. This is based on the observation in the Lund cohort that the majority of women who experienced a second PID did so within 2 years. To allow the rate of progression to change with time since last episode the model includes eight states and women may transition between these states as described in Figure 1. The model does not consider what happens to women who have $>3$ episodes because data on the risk distribution of sequelae, and external validation data, do not distinguish between such women. The key outputs from each model are the proportions of women in age group $a$ in the general population who have experienced $i=0,1,2, \geqslant 3$ previous episodes or diagnosed episodes. These are simple functions of the model parameters (see Supplementary material 1).

\section{Model 1: distribution of clinical PID in the English general population}

In this model episodes represent clinical PID. We make the assumption that the ratio of the incidence rate of PID in women who have had a PID in the last 2 years to women who have not is independent of age. We performed a literature search to identify all relevant sources of evidence for the model parameters and functions of the model parameters in England [9] (Supplementary material 2). Note that these estimates are correlated because they are estimated from the same data, and are therefore incorporated into the Markov model as a multivariate lognormal likelihood with covariances calculated from the synthesis model.

\section{Model 2: distribution of salpingitis in the English population}

In this model an episode represents salpingitis. We make the assumptions that the proportion of clinical PID episodes that are salpingitis is independent of age and PID/salpingitis history. Note that in this 


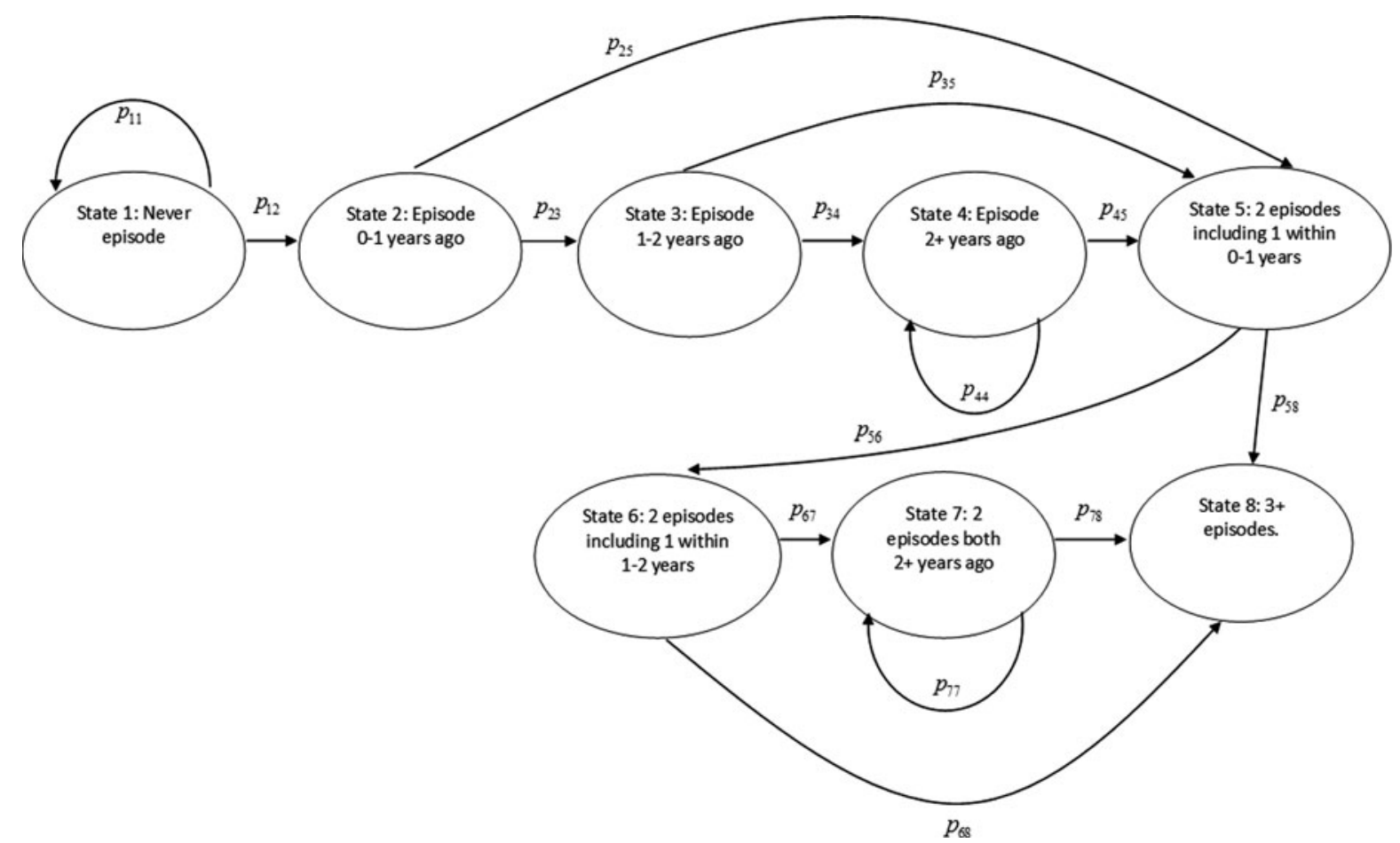

Fig. 1. Markov progression model of disease incidence. The definition of an 'episode' differs between models. Individuals start in state 1 and can progress to state 2. Once in state 2, women can either fail to have an episode and enter state 3 after one cycle or have an episode and enter state 5 . From state 3 women can either fail to have an episode and enter state 4 after one cycle or have an episode and enter state 5 . The progression pattern between states 5 and 8 mirrors that between states 1 and 4; state 8 is an absorbing state.

model the higher rate only applies to women who have had a salpingitis episode within the previous 2 years and not women who have had non-salpingitis PID. In addition to the data described above we require information on the proportion of clinical PID episodes that are salpingitis (see Supplementary material 2).

\section{Model 3: distribution of PID in women with salpingitis history in the English population}

A final model considers the proportion of women in England by age who have had at least one salpingitis episode, and the number of subsequent clinical PID episodes, i.e. $0,1, \geqslant 2$, that they have had. Therefore the first episode is salpingitis and the second and third episodes are PID. This model produces estimates that are comparable to the form of the data from the Lund studies allowing external validation of the model against these data.

\section{Methodology for estimation and computation}

Estimation is performed with a Bayesian approach using Markov chain Monte Carlo (MCMC) simulation in WinBUGS v. 1.4.3 [15] and the addon package WBDev. Posterior means and 95\% credible intervals for parameters and model outputs are reported. This method ensures that all the uncertainty in the data and estimates for all parameters is fully propagated into the model outputs. Unless otherwise stated vague priors are employed throughout, so that results are dominated by the data. Further details are given in Supplementary material 3.

\section{Summary of assumptions}

We have made the following key assumptions

(1) The incidence rate for PID is the same within age groups 16-19, 20-24, 25-34 and 35-44 years.

(2) The incidence rate for PID in women aged $<16$ years is zero.

(3) Women who have a PID episode have a different (higher) rate of subsequent PID episodes for the next 2 years and the ratio of these rates is independent of age. Furthermore, the pattern of infection and re-infection in CT, is the same as the pattern of PID and repeat PID, for PID from any cause. 
(4) Conditional upon assumption (3), PID incidence is independent of PID history.

(5) Estimated incidence of diagnosed PID from routine data sources is uniformly distributed between the total observed in STI clinics + the maximum from hospital episode statistics (HES) and scaled GPRD data and the total from STI, HES, and scaled GPRD data.

(6) The probability that a clinical PID episode is diagnosed is independent of age and PID history.

(7) The probability that a clinical PID episode is salpingitis is independent of age and PID/salpingitis history.

\section{External validation}

We validated our results against the Lund study [2], which reports data on the distribution of numbers of PID episodes in women who have had salpingitis, for a mean follow-up period of $\sim 8$ years, separately for women aged under and over 25 years. The Markov model was run separately for women starting at each of the 22 1-year age bands (16-37 years), in each case starting in state 2 , for an 8-year time-horizon. The average predicted number of women with a single, and $\geqslant 2$ subsequent PID episodes were obtained by averaging across the age ranges 16-24, and 25-37 years, respectively. Results for all PID and diagnosed PID only are shown alongside the Lund data (Table 1).

\section{Sensitivity analysis}

We developed a fully probabilistic model which accounts for all of the uncertainty in the data that has been used to estimate the parameters. However, the sensitivity of the results to some key structural assumptions are assessed in Supplementary material 4.

\section{RESULTS}

Table $2 a$ gives the predicted numbers of women who have had $0,1,2$, or $\geqslant 3$ previous PID episodes, whether diagnosed or not, by age. Exactly analogous sets of predictions are shown for diagnosed PID (Table $2 b$ ) and for salpingitis (Table 2c). These tables show that $33.6 \%$ of women aged 25-44 years have experienced at least one episode of PID, and that $16 \cdot 1 \%$ have experienced at least one episode of salpingitis, again all-cause and whether diagnosed or not. Finally Table $2 d$ shows the proportions of the population that have experienced at least one episode of salpingitis, followed by 0,1 , or $\geqslant 2$ episodes of PID. Here we see that while $16 \cdot 1 \%$ have experienced at least one episode of salpingitis, $10 \cdot 7 \%$ have experienced one salpingitis episode and no further PID episodes, $3.7 \%$ one salpingitis and one further PID episode, and $1.7 \%$ one salpingitis and $\geqslant 2$ further PID episodes. Figure 2 gives essentially the same results, respectively, in 1-year bands from ages 16 to 44 years.

\section{External validation}

Table 1 shows the correspondence between the results from the Markov model run for an 8-year period and the Lund data. The comparisons between observed and predicted distributions only concern the proportions of PIDs that are second or third (or more) PID episodes, because only women who have a PID were recruited into the Lund study. Note that there is no reason to expect the credible intervals to agree. The first column shows the proportions of women in the study that the model predicts would develop 1, or $\geqslant 2$ PIDs, whether diagnosed or not, during the follow-up period. Column 2 shows how many PIDs would be expected to be observed (diagnosed) in these women. The Lund study results (column 3) lie between the results in the first two columns, which is exactly what is to be expected as it seems reasonable that subsequent PID episodes in women who have had a previous, relatively recent, hospital diagnosed PID are more likely than average to be diagnosed, on the basis that (i) these women will be more likely to recognize the symptoms, and (ii) such PIDs may be more severe than average. On the other hand, the Lund study is not technically a cohort study: unlike the POPI trial participants, women recruited into the Lund study would not have been told specifically to look out for symptoms, and the follow-up time was much longer, so we would not necessarily expect all, or even most, symptomatic PID to be diagnosed.

\section{DISCUSSION}

This paper proposes, as far as we are aware for the first time, a methodology for estimating the proportion of incident PID episodes, and salpingitis episodes, that are first, second, or third episodes, by age. The method is based on assumptions about the CT re-infection to infection rate ratio, and the length of time after which the re-infection rate applies. Under these 
Table 1. Distribution of numbers of PID after 8 years, posterior mean $\%$ in each category (95\% credible intervals), compared to the findings from the Lund study (see text)

\begin{tabular}{lccc}
\hline \hline No. of episodes & Model: all PID & Model: diagnosed PID & Lund study \\
\hline Age 16-24 years & & & \\
1 PID & $66 \cdot 3(51 \cdot 3-78 \cdot 0)$ & $85 \cdot 4(78 \cdot 3-90 \cdot 2)$ & $77 \cdot 7(75 \cdot 1-80 \cdot 3)$ \\
2 PID & $23 \cdot 6(18 \cdot 3-27 \cdot 2)$ & $12 \cdot 8(9 \cdot 1-17 \cdot 8)$ & $16 \cdot 0(13 \cdot 8-18 \cdot 4)$ \\
3 PID & $10 \cdot 1(3 \cdot 75-21 \cdot 5)$ & $1 \cdot 8(0 \cdot 7-3 \cdot 9)$ & $6 \cdot 2(4 \cdot 8-7 \cdot 8)$ \\
Age 25-44 years & $75 \cdot 2(62 \cdot 3-84 \cdot 7)$ & $90 \cdot 0(84 \cdot 4-93 \cdot 5)$ & $87 \cdot 0(82 \cdot 6-90 \cdot 9)$ \\
1 PID & $19 \cdot 3(13 \cdot 5-25 \cdot 0)$ & $9 \cdot 3(6 \cdot 2-13 \cdot 6)$ & $11 \cdot 0(7 \cdot 5-15 \cdot 1)$ \\
2 PID & $5 \cdot 5(1 \cdot 8-12 \cdot 7)$ & $0 \cdot 9(0 \cdot 3-2 \cdot 0)$ & $2 \cdot 0(0 \cdot 6-4 \cdot 0)$ \\
$\geqslant 3$ PID & &
\end{tabular}

PID, Pelvic inflammatory disease.

Comparison between observed and predicted proportions is only relevant for PIDs that are second or third (or more) PID episodes - the numbers not in italics (see text).

assumptions, the results are compared to data on the distribution of second and third episodes in women with an index episode. We used a variety of high-quality data sources to estimate the parameters and where possible assessed their consistency. The impact of key structural assumptions was assessed in sensitivity analysis.

In the Lund study $22 \cdot 1 \%$ of women aged 16-24 years with an index salpingitis episode were observed to have a further PID episode within 8 years, and $6.2 \%$ had more than one further episode. In women aged $25-44$ years $11 \%$ had a further PID episode, and $2 \%$ more than one. The Lund study follows women whose index PID episode was sufficiently severe to be diagnosed and treated in hospital. So our estimate of the proportion of PIDs that are diagnosed in the general population is likely to be lower than the proportion in the Lund dataset. Therefore our external validation with this data must be informal. All we can say is that the Lund results should lie somewhere between our estimates for the numbers of subsequent PIDs and the numbers of subsequent PIDs likely to be diagnosed in the general population.

Within year and diagnostic pathway repeat PID rates are available for hospital and GUM settings $[16,17]$. Annually, $3 \cdot 2-3 \cdot 4 \%$ of PID cases recorded in HES are within-year repeat cases. The population at risk of a first annual HES diagnosis is far higher than for a second or subsequent diagnosis, so the HES PID diagnosis rate is nearly 20 -fold higher in the latter. GUM data are similar. This is higher than our base-case average rate ratio which covers a 2-year period, and slightly higher than our sensitivity analysis. So we may have overestimated ever PID and underestimated repeat episodes. However, it is unknown how long this repeat case rate persists past 6 months (average).

We use data from the UK study by TaylorRobinson to estimate the proportion of PID cases that are salpingitis [18]. Although the study was published fairly recently the data were collected in the 1990s and cases were diagnosed in hospital, so it is unclear how applicable it is to all PID in 2002. Over time, clinical guidance has changed recommending treating women with possible or probable PID instead of only treating women with probable PID so this is likely to be an overestimate for the proportion in all PIDs in 2002. Although we only included PID cases from the GPRD database that were definite or probable it is unclear whether the Taylor-Robinson study provides an overestimate for clinical PID cases diagnosed in GUM clinics. We also assume the same proportion of salpingitis in undiagnosed PID cases and there is no real evidence to say whether this is reasonable. On the one hand, undiagnosed women are likely to have less severe symptoms and symptoms are likely to correlate to severity of inflammation and presence of salpingitis. Howover, laparoscopy identifies the presence of salpingitis at a single point in time. Some of the women in the Taylor-Robinson study may have developed inflammation that would be visible on laparoscopy at a later date had they not been treated as would be the case if they were undiagnosed.

Our estimates of cumulative incidence of diagnosed PID are considerably higher than the National Survey of Sexual Attitudes and Lifestyles (Natsal) [19] which reported that $2 \cdot 2 \%(95 \%$ confidence interval $1 \cdot 8-2 \cdot 6 \%)$ of female respondents aged 16-44 years said they have ever been treated for PID compared to our estimate of $\sim 10 \%$ in 31-year-olds (Fig. 2). However, Natsal is also 
Table 2. Predicted age-specific distribution of numbers of previous episodes from the Markov model. Posterior mean \% in each category (95\% credible intervals)

\begin{tabular}{|c|c|c|c|c|}
\hline \multicolumn{5}{|l|}{ (a) PID } \\
\hline Age, years & 0 PID episode & 1 PID episode & 2 PID episodes & $\geqslant 3$ PID episodes \\
\hline \multicolumn{5}{|l|}{ All PID } \\
\hline $16-19$ & $95 \cdot 7(94 \cdot 2-96 \cdot 9)$ & $3 \cdot 89(2 \cdot 90-5 \cdot 09)$ & $0 \cdot 43(0 \cdot 19-0 \cdot 81)$ & $0 \cdot 03(0 \cdot 01-0 \cdot 10)$ \\
\hline $20-24$ & $86 \cdot 7(82 \cdot 5-90 \cdot 2)$ & $10 \cdot 3(7 \cdot 94-12 \cdot 9)$ & $2.38(1.25-3.91)$ & $0.64(0 \cdot 16-1 \cdot 69)$ \\
\hline $25-34$ & $75 \cdot 3(67 \cdot 9-81 \cdot 6)$ & $17 \cdot 2(13 \cdot 7-21 \cdot 0)$ & $5 \cdot 30(3 \cdot 10-7 \cdot 95)$ & $2 \cdot 16(0 \cdot 64-5 \cdot 25)$ \\
\hline $35-44$ & $66 \cdot 4(56 \cdot 9-74 \cdot 6)$ & $22 \cdot 2(18 \cdot 2-26 \cdot 3)$ & $7 \cdot 77(4 \cdot 83-11 \cdot 1)$ & $3 \cdot 67(1 \cdot 21-8 \cdot 49)$ \\
\hline \multicolumn{5}{|c|}{ (b) Diagnosed PID } \\
\hline Age, years & 0 PID episode & 1 PID episode & 2 PID episodes & $\geqslant 3$ PID episodes \\
\hline \multicolumn{5}{|l|}{ All PID } \\
\hline $16-19$ & $98 \cdot 4(98 \cdot 2-98 \cdot 5)$ & $1.58(1.41-1.75)$ & $0 \cdot 06(0 \cdot 03-0 \cdot 10)$ & $0 \cdot 00(0 \cdot 00-0 \cdot 00)$ \\
\hline $20-24$ & $94 \cdot 6(93 \cdot 8-95 \cdot 2)$ & $4.97(4 \cdot 47-5 \cdot 50)$ & $0 \cdot 44(0 \cdot 26-0 \cdot 70)$ & $0.03(0.01-0.06)$ \\
\hline $25-34$ & $89 \cdot 3(87 \cdot 6-90 \cdot 7)$ & $9 \cdot 44(8 \cdot 36-10 \cdot 6)$ & $1 \cdot 15(0 \cdot 73-1 \cdot 75)$ & $0.09(0.04-0.18)$ \\
\hline $35-44$ & $84 \cdot 7(82 \cdot 3-86 \cdot 8)$ & $13 \cdot 0(11 \cdot 6-14 \cdot 6)$ & $1 \cdot 81(1 \cdot 22-2 \cdot 64)$ & $0 \cdot 49(0 \cdot 31-0 \cdot 76)$ \\
\hline \multicolumn{5}{|c|}{ (c) Salpingitis } \\
\hline Age, years & 0 salpingitis episode & 1 salpingitis episode & 2 salpingitis episodes & $\geqslant 3$ salpingitis episodes \\
\hline \multicolumn{5}{|c|}{ All salpingitis } \\
\hline $16-19$ & $98 \cdot 1(97 \cdot 0-99 \cdot 0)$ & $1 \cdot 85(1 \cdot 02-2 \cdot 88)$ & $0 \cdot 04(0 \cdot 01-0 \cdot 14)$ & $0 \cdot 00(0 \cdot 00-0 \cdot 00)$ \\
\hline $20-24$ & $94 \cdot 1(90 \cdot 7-96 \cdot 8)$ & $5 \cdot 56(3 \cdot 18-8 \cdot 35)$ & $0.34(0.07-0.93)$ & $0 \cdot 02(0 \cdot 00-0 \cdot 10)$ \\
\hline $25-34$ & $88 \cdot 6(82 \cdot 3-93 \cdot 6)$ & $10 \cdot 4(6 \cdot 11-15 \cdot 0)$ & $0.99(0.23-2.44)$ & $0 \cdot 10(0 \cdot 01-0 \cdot 40)$ \\
\hline $35-44$ & $83 \cdot 9(75 \cdot 3-91 \cdot 0)$ & $14 \cdot 2(8 \cdot 55-20 \cdot 1)$ & $1 \cdot 71(0 \cdot 45-3 \cdot 96)$ & $0 \cdot 20(0 \cdot 02-0 \cdot 78)$ \\
\hline \multicolumn{5}{|c|}{ (d) Salpingitis and subsequent PID } \\
\hline Age, years & 0 salpingitis episode & $\begin{array}{l}1 \text { salpingitis, } 0 \text { further } \\
\text { PID episode }\end{array}$ & $\begin{array}{l}1 \text { salpingitis, } 1 \text { further } \\
\text { PID episode }\end{array}$ & $\begin{array}{l}1 \text { salpingitis, } \geqslant 2 \text { further } \\
\text { PID episodes }\end{array}$ \\
\hline \multicolumn{5}{|l|}{ All cause } \\
\hline $16-19$ & $98 \cdot 1(97 \cdot 0-99 \cdot 0)$ & $1.69(0.93-2.64)$ & $0 \cdot 19(0 \cdot 07-0 \cdot 38)$ & $0 \cdot 01(0 \cdot 00-0 \cdot 04)$ \\
\hline $20-24$ & $94 \cdot 1(90 \cdot 7-96 \cdot 8)$ & $4.59(2.59-6.99)$ & $1.06(0 \cdot 46-1.96)$ & $0 \cdot 28(0 \cdot 06-0 \cdot 79)$ \\
\hline $25-34$ & $88 \cdot 6(82 \cdot 3-93 \cdot 6)$ & $8 \cdot 03(4 \cdot 63-12 \cdot 0)$ & $2 \cdot 44(1 \cdot 15-4 \cdot 21)$ & $0 \cdot 98(0 \cdot 25-2 \cdot 55)$ \\
\hline $35-44$ & $83 \cdot 9(75 \cdot 3-91 \cdot 0)$ & $10 \cdot 7(6 \cdot 32-15 \cdot 7)$ & $3 \cdot 66(1 \cdot 80-6 \cdot 14)$ & $1 \cdot 69(0 \cdot 48-4 \cdot 18)$ \\
\hline
\end{tabular}

PID, Pelvic inflammatory disease. 
(a) PID

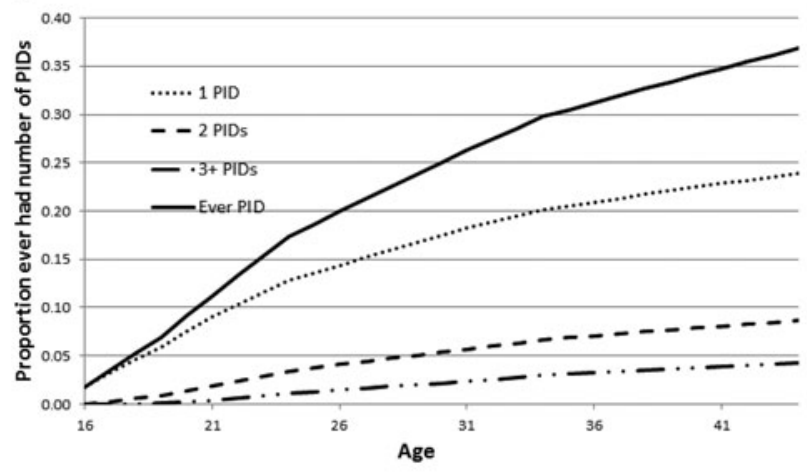

(c) Salpingitis

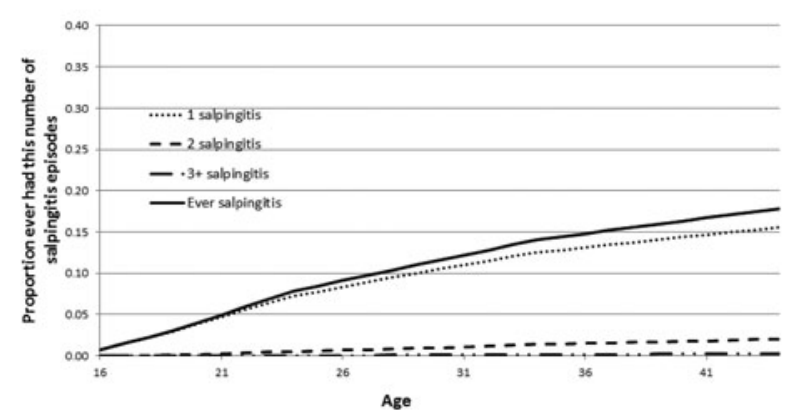

(b)

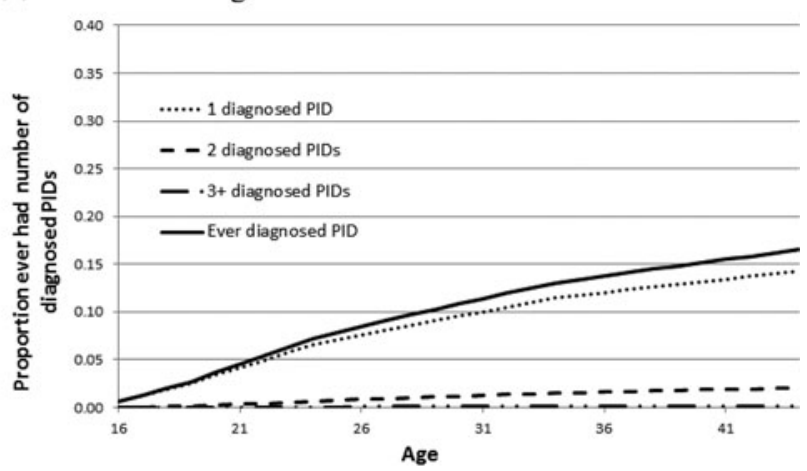

(d)

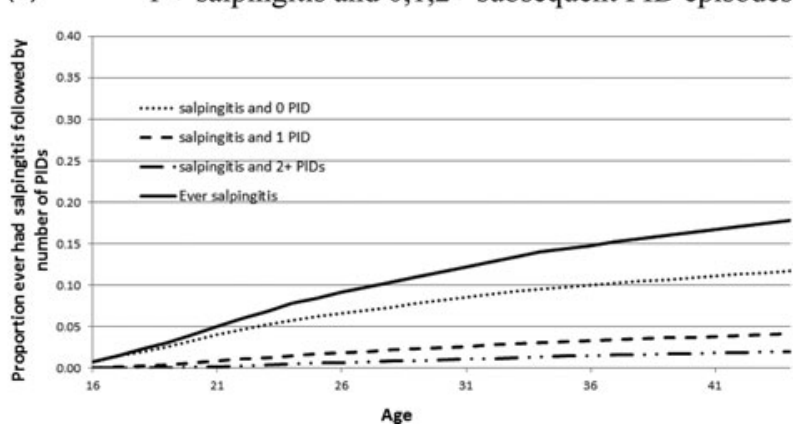

Fig. 2. Cumulative exposure: proportion experiencing $1,2, \geqslant 3$ episodes by age (years).

highly inconsistent with other UK data sources. The POPI trial [7] observed all-cause PID incidence to be $\sim 2 \%$ in a single year. Furthermore, HES data alone report a total of $\sim 15000$ PIDs in women by the age of 35 years. If the 20 -fold repeat case PID rate persists throughout a woman's reproductive life after diagnosis this would be consistent with Natsal. But this would be at odds with the Lund data and does not consider GP or GUM diagnoses. Recruitment and participation biases in surveys like Natsal may selectively under-sample those who would be considered at increased risk (and some groups at reduced risk). On top of this there may be a tendency in responders to under-report health problems linked to sexually transmitted disease, and it may be that not everyone diagnosed with PID is told this diagnosis and remembers it. In fact GPRD codes for PID often do not mention PID specifically. The discrepancy between Natsal and our results is, nevertheless, large and requires further investigation.

Our projections can also be compared with the 2002 US National Survey of Family Growth in which $5 \cdot 1 \%$ of women aged 16-44 years reported having been treated for PID [20]. This figure is sharply down on the 1995 survey which reported $8 \%$ had been treated for PID, with $11 \%$ in the 1988 and $14 \%$ in the 1982 surveys. Our average estimate for this age range is around $10 \%$. In the Uppsala study, the cumulative incidence of hospital-diagnosed PID was reported as $3.9 \%$ by 35 years [21]. If we use only HES data to estimate the incidence rate of diagnosed PID, the estimate of cumulative incidence in women aged 35 years is $4 \cdot 6 \%$, close to the Uppsala figure.

The population-level results are primarily applicable to England in 2002 just before the introduction of the National Chlamydia Screening Programme (NCSP) [22], so the estimates can be used in conjunction with epidemiological studies of natural history. However, the model could easily be applied to other countries or times provided the necessary data were available to fit it.

\section{CONCLUSIONS}

We estimate that in England in 2002, 33.6\% of women aged 35-44 years had experienced at least one episode of PID (diagnosed or not) and $16 \cdot 1 \%$ of them had experienced at least one episode of salpingitis (diagnosed or not). Further work is required to assess the degree of overlap between routine data sources for PID and of PID history for women diagnosed with PID. Linkage of routine data sources would enable 
tracking of referrals between settings, and ideally over time. Cross-sectional or retrospective studies of the proportion of PID that is diagnosed, and the proportion of PIDs that are salpingitis together with the severity distribution in different diagnostic settings would be valuable.

\section{SUPPLEMENTARY MATERIAL}

For supplementary material accompanying this paper visit http://dx.doi.org/10.1017/S0950268816002065.

\section{ACKNOWLEDGEMENTS}

The work was supported by UK Medical Research Council Project Grant no. G0801947.

\section{DECLARATION OF INTEREST}

P.J.H. reports receiving personal fees from Aquarius Population Health; grants, personal fees and nonfinancial support from Cepheid; personal fees from Crown Prosecution Service; personal fees from British Association for Sexual Health and HIV; grants from Mast Group Ltd; grants and personal fees from Hologic, outside the submitted work; in addition P.J.H. has a patent A sialidase spot test to diagnose bacterial vaginosis, issued to the University of Bristol. The remaining authors report no conflicts of interest.

\section{REFERENCES}

1. Paavonen J, Westrom L, Eschenbach DA. Pelvic inflammatroy disease. In Sexually Transmitted Disease. In: Holmes K, Sparling PF, Stamm WE, eds. London: McGraw Hill, 2008, pp. 1021-1022.

2. Westrom $\mathbf{L}$, et al. Pelvic inflammatory disease and fertility - a cohort study of 1,844 women with laparoscopically verified disease and 657 control women with normal laparoscopic results. Sexually Transmitted Diseases 1992; 19: 185-192.

3. Brunham RC, Gottlieb SL, Paavonen J. Pelvic inflammatory disease. New England Journal of Medicine 2015; 372: 2039-2048.

4. British Association for Sexual Health and HIV Clinical Effectiveness Group. UK national guidline for the management of pelvic inflammatory disease 2011. British Association for Sexual Health and HIV 2011, p. 18.

5. Hager W, Eschenbach D, Spence M. Criteria for diagnosis and grading of salpingitis. Obstetrics and Gynecology 1983; 61: 113-14.
6. Taylor-Robinson D, et al. Further observations, mainly serological, on a cohort of women with or without pelvic inflammatory disease. International Journal of STD \& AIDS 2009; 20: 712-718.

7. Oakeshott P, et al. Randomised controlled trial of screening for Chlamydia trachomatis to prevent pelvic inflammatory disease: the POPI (prevention of pelvic infection) trial. British Medical Journal 2010; 340: c1642.

8. French CE, et al. Estimation of the rate of pelvic inflammatory disease diagnoses: trends in England, 2000 2008. Sexually Transmitted Diseases 2011; 38: 158-162.

9. Price M, et al. The natural history of Chlamydia trachomatis infection in women: a multi-parameter evidence synthesis. Health Technology Assessment Methodology report (in press).

10. Wolner-Hanssen P. Silent pelvic inflammatory disease is it overstated? Obstetrics and Gynecology 1995; 86: 321-325.

11. Ross JMG. UK national guideline for the management of pelvic inflammatory disease, 2011.

12. Hillis SD, et al. Delayed care of pelvic inflammatory disease as a risk factor for impaired fertility. American Journal of Obstetrics and Gynecology 1993; 168: 15031509.

13. Westrom L, Bengtsson LP, Mardh PA. Incidence, trends, and risks of ectopic pregnancy in a population of women. British Medical Journal 1981; 282: 15-18.

14. Westrom LV. Sexually-transmitted diseases and infertility. Sexually Transmitted Diseases 1994; 21: S32-S37.

15. Lunn DJ, et al. WinBUGS - a Bayesian modelling framework: concepts, structure, and extensibility. Statistics and Computing 2000; 10: 325-337.

16. Hospital Episode Statistics (HES). Re-used with the permission of the Health and Social Care Information Centre, 2016.

17. Public Health England. Genitourinary medicine clinic activity dataset (version 2) (GUMCADv2), 2016.

18. Taylor-Robinson D, et al. Difficulties experienced in defining the microbial cause of pelvic inflammatory disease. International Journal of STD \& AIDS 2012; 23: 18-24.

19. Fenton KA, et al. Sexual behaviour in Britain: reported sexually transmitted infections and prevalent genital Chlamydia trachomatis infection. Lancet 2001; 358: 1851-1854.

20. Chandra A, et al. Fertility, family planning, and reproductive health of U.S. women: data from the 2002 National Survey of Family Growth. National Center for Health Statistics. Vital Health Statistics 2005; 23: $1-160$.

21. Low N, et al. Incidence of severe reproductive tract complications associated with diagnosed genital chlamydial infection: the Uppsala Women's Cohort Study. Sexually Transmitted Infections 2006; 82: 212-218.

22. Public Health England. National Chlamydia Screening Programme (http://www.chlamydiascreening.nhs.uk/ps/ index.asp). 2014 